\title{
Conflictos POR la Herencia DE LA TIERRA EN UNA FAMilia DE ChENALHO': UNA VOZ DIVIDIDA
}

\section{Conflicts over the Land Inheritance in One Family From Chenalho': A Divided Voice}

\author{
Elías Pérez-Pérez* \\ Witold Jacorzynski**
}

Resumen: En este artículo se examinan las prácticas de herencia en una familia tsotsil del municipio de Chenalho' en Los Altos de Chiapas. Se argumenta que la unidad básica de la herencia se basa en prácticas sociales específicas, con contextos, reglas, estilos y discursos, y que los conflictos por la herencia refuerzan el liderazgo del padre y del hermano mayor en la familia patriarcal. El método de exposición es dialógico y perspectivista. En la primera parte un narrador relata la historia de los conflictos en su familia, y en la segunda otro narrador analiza lo expuesto por el primero. En conclusión, se muestran y analizan los principios por los que se rige la herencia en Chenalho'.

Palabras clave: herencia de la tierra, conflictos intrafamiliares, familia, patriarcado.

Abstract: This paper describes and analyses the inheritance practices among the members of one Tsotsil family from Chenalhó, in the Highlands of Chiapas. We argue that, first, the basic unit of social life is practices with their actors, contexts, rules, styles, and discourses; and, second, the conflicts over inheritance in the family contribute to maintaining the leadership of the father and eldest brother within a patriarchal family. The exposition has a dialogical and perspectivist structure. This paper is divided into two parts: In the first, a narrator tells the story of his family's conflicts, and in the second, a different narrator analyses the conflict from his own perspective. Finally, the rules governing inheritances are made transparent and analyzed.

Keywords: land inheritance, intra-family conflicts, family, patriarchy.

\footnotetext{
* Elías Pérez Pérez. Doctor en Antropología por la Universidad de Salamanca, España. Profesor-investigador de la Universidad Politécnica Nacional, México. Temas de especialización: educación indígena, pueblos indígenas. Correo electrónico: elias_perez28@hotmail.com. ORCID: https://orcid.org/0000-0001-6887-0238.

** Witold Jacorzynski. Doctor en Ciencias Humanísticas por la Universidad de Varsovia Polonia. Profesor-investigador del CIESAS Sureste, México. Temas de especialización: epistemología, pueblos indígenas, ética antropológica. Correo electrónico: lekvinik64@gmail. com. ORCID: https://orcid.org/0000-0002-9784-5653.
}

Enviado a dictamen: 19 de febrero de 2018

Aprobación: 4 de septiembre de 2018 Revisiones: 1 


\section{Introducción}

E ste trabajo surgió a raíz de nuestro interés por las prácticas de herencia en Chenalho'. Este tema, tan importante como olvidado, ha sido analizado con mayor detalle en los municipios de Zinacantán (Collier, 1976) o San Juan Chamula (Contreras 2001, 2003). Las monografías más importantes sobre Chenalho' mencionan dichas prácticas sin profundizar en ellas (Guiteras, 1996; Arias, 1975, 1985; Garza, 2002, 2007). Una excepción notable a esta regla es el trabajo de Ixtacuy, Estrada y Parra (2006), aunque en él brilla por su ausencia el lenguaje emic y el punto de vista del "nativo". Se espera que el presente ensayo, escrito tanto en lenguaje emic como etic, pueda completar el análisis de las prácticas sociales en torno a la herencia de los bienes en Chenalho'.

El primer objetivo de este ensayo es describir las prácticas de la herencia tomando como ejemplo una familia de la comunidad Las Limas del municipio señalado. Dicha familia pertenece al linaje de los Pasinsa, actores de la conversión colectiva a la nueva fe presbiteriana en los años cincuenta del siglo pasado. Uno de los líderes de esa conversión fue Victorio Pasinsa, el sexto hijo de Antún Pasinsa, antiguo líder tradicionalista. Victorio emigró a Las Limas de Chibtik, la comunidad natal de Antún, en el año 1970. Nuestra historia versa sobre las prácticas de repartición de la tierra y otros bienes de Victorio entre sus hijos e hijas en diferentes periodos: desde el primer reparto a finales de los años setenta del siglo pasado, pasando por un segundo tras su muerte el 28 de mayo de 2016, y un tercero efectuado a finales de julio de 2017.

El segundo objetivo es aportar nuevas reflexiones sobre la herencia a partir de la filosofía de las prácticas de Ludwig Wittgenstein y la teoría del conflicto de George Simmel, sociólogo alemán de la primera mitad del siglo XX. Mientras que la filosofía del lenguaje de Wittgenstein asume que la vida social consta de los juegos de lenguaje, prácticas o actividades (Wittgenstein, 1988), la idea principal de Simmel es que los conflictos entre las prácticas, o al interior de ellas, cumplen un papel no sólo negativo sino, en ciertas ocasiones, positivo (Simmel, 2009: 227-306). En nuestra descripción partiremos de casos conflictivos en el proceso de heredar la tierra, no tanto de casos comunes, porque valoramos que el estudio del conflicto tiene dos ventajas; en primer lugar, sugerimos que sólo a partir del conflicto se reproduce la norma y, en segundo lugar, consideramos que el conflicto puede mostrar no sólo las características de la contienda, sino, además, las diferentes maneras de solución.

La presentación de los datos se dividirá en dos narrativas. En la primera parte, el autor, miembro del linaje Pasinsa, hablará de los conflictos en el proceso de heredar la tierra en su familia. Posteriormente, el segundo narrador tomará la batuta para resumir y analizar el relato del primer autor.

\section{Prácticas y conflictos: reflexiones teóricas}

Para identificar y describir las prácticas de herencia deben responderse cinco preguntas básicas: 1) iqué se entiende por diferentes prácticas de heredar?, 2) ¿en qué circunstancias, cuándo y dónde se hereda? 3) ¿quiénes son los actores que participan en las actividades relacionadas con la herencia?, 4) icuáles son las intenciones, los intereses y las estrategias de los practicantes?, 5) icómo o según qué reglas se hereda? y 6) ¿cómo se justifican las prácticas de herencia?

La primera pregunta es fundamental y a ella puede responderse haciendo referencia a juegos de lenguaje y a prácticas relacionadas con "heredar". Siguiendo a Wittgenstein, entendemos por juego de lenguaje "al todo formado por el lenguaje y las acciones con las que está entretejido" (Wittgenstein, 1988: párr. 7). "Herencia" es una palabra general que abarca un conglomerado de prácticas vinculadas como: la medición del terreno en brazadas y tareas, reuniones familiares para discutir casos problemáticos y reparto de cosas en el momento del matrimonio. Este conjunto de prácticas está anclado en un todo más grande: la forma de vida; se "hereda la tierra" o "se reparte la tierra" perteneciente hasta entonces al padre. No toda 
la tierra adquirida durante la vida de la persona forma parte de la herencia paterna, porque sólo la tierra que la persona compra durante su vida forma parte de la herencia de sus hijos.

La segunda pregunta invita a reflexionar acerca de la situación, el tiempo y el espacio en que se lleva a cabo la repartición de la tierra, circunstancias que dependen de la forma de vida y del contexto. No sería difícil imaginar una sociedad en la cual no se llevaran a cabo repartos de tierra, una sociedad en la cual la tierra no fuera entendida como un bien divisible, donde no existiesen los conceptos de propiedad ni de herencia, ni el concepto de "padre", ni el de vida sedentaria; en sociedades de esta naturaleza no sería posible "heredar la tierra", y esta imposibilidad no sería empírica, sino conceptual.

Las preguntas tercera y cuarta hacen referencia a los actores que participan en las actividades de herencia, y a sus intenciones, intereses y estrategias. Es importante relacionar estas preguntas con la quinta, que hace referencia a las reglas que rigen esta práctica, lo cual es fundamental para quienes buscan la reconciliación entre la estructura y la agencia. La solución wittgensteiniana a este problema es la siguiente: cada práctica está regida por reglas, pero ninguna práctica está totalmente delimitada por ellas; por ejemplo, aunque el tenis tenga reglas estrictas, ninguna dice cuán alto o cuán fuerte se puede lanzar la pelota (Wittgenstein, 1988: párr. 68). De esta manera, la acción social se encuentra en función de las reglas y los estilos individuales que "llenan" la esfera no reglamentada o ambigua del juego. En el caso concreto de la herencia de la tierra, proponemos como marco de referencia la subjetividad del heredero, su papel social y sus intereses, porque el significado de lo que hacen y dicen los protagonistas se revela en sus flujos de vida.

La quinta pregunta, que se refiere a las reglas según las cuales se desenvuelve la práctica de heredar, evoca sobre todo lo que Wittgenstein llamaba "bisagras" o "reglas de control". Estas últimas constituyen el weltbild, la imagen del mundo, la cual forma una red: "No me aferro a una proposición, sino a una red de proposiciones" (Wittgenstein, 1997: párr. 225). En este sentido: "Cuando empezamos a creer algo, lo que creemos no es una única proposición sino todo un sistema de proposiciones. (Se hace la luz poco a poco sobre el conjunto)" (Wittgenstein, 1997: párr. 141). Esta pregunta es compleja y a la vez seductora, pues invita a clasificar estas reglas en, por ejemplo, lingüísticas, personales, universales y culturales (Moyal-Sharrock, 2004). En lugar de ofrecer una lista de tales reglas analizaremos primero algunos ejemplos de conflictos relacionados con la herencia de la tierra para hacer visibles las normas que se han violado.

En cuanto a la última pregunta, relacionada con cómo se justifican las prácticas que se llevan a cabo, desde el inicio debe contemplarse que las justificaciones, explicaciones o apologías forman parte de los discursos, los cuales se vuelven importantes únicamente cuando las prácticas son objeto de crítica o controversia (Clooney, 2005). Mientras que tanto las prácticas como sus justificaciones cambian con el tiempo, estas últimas se ajustan más al ritmo de los cambios y son más volátiles y flexibles. Las prácticas son los fundamentos sobre los cuales se edifican los discursos. La adaptación de los discursos nuevos a las viejas prácticas pasan por un proceso de adaptación que suele llamarse "crisis gramsciana": las prácticas viejas están obsoletas, pero no existen aún palabras para hacer referencia a las prácticas que están por nacer: "La crisis consiste justamente en que lo viejo muere y lo nuevo no puede nacer" (Gramsci 1990: 52).

El punto de partida del presente estudio es el análisis de los conflictos por la tierra; en este sentido, nos proponemos estudiar los conflictos y no las prácticas simpliciter principalmente por dos razones. La primera es en parte conceptual y en parte metodológica. Si cuando se pone en práctica una acción se sigue una regla, icómo descubrir tal regla? La primera manera de hacerlo sería observando casos similares para inducir la conducta "normal" o típica que suele llevarse a cabo. Esta modalidad es onerosa, innecesariamente larga y metodológicamente desalentadora, porque la regla se descubre de manera más fácil a partir de relatos sobre los errores que se han producido al seguirla que a partir de los casos en los 
que se sigue de forma correcta. Como escribe Winch: "La noción de seguir una regla es inseparable de la noción de cometer un error" (Winch, 1990: 35). Para este autor, no es el individuo aislado quien se percata de cuándo ha cometido un error, y por lo tanto se ha desviado de una regla, sino es la comunidad la que corrige al individuo errado: "El hecho de establecer una norma no es una actividad que se puede adscribir a cualquier individuo completamente aislado de otros, ya que sólo un contacto con estos últimos posibilita control de las propias acciones, control inseparable de una pauta establecida" (Winch 1990: 35). Como se analizará más adelante, las reglas de herencia no se siguen ni automática ni individualmente, sino que los participantes en esta práctica forman una pequeña comunidad que controla los pasos de los individuos.

La segunda razón es de índole más sustancial y teórica. El “conflicto” es un fenómeno sociológicamente más importante que la concordancia, idea que defendió Georg Simmel:

El malentendido según el cual lo que uno construye el otro lo echa a perder y lo que queda es el resultado de la diferencia entre los dos (mientras que en la realidad se puede identificar mejor con la suma de los dos) se origina, probablemente, en el doble significado del concepto de unidad. Comprendemos por unidad el consenso y la combinación de los elementos sociales en contraste con sus divisiones, disociaciones, discrepancias; la unidad, sin embargo, refiere también a la síntesis de personas, energías y formas en grupos, la totalidad final de ellas, en la cual están incluidas tanto la integridad, en el sentido más estricto, como las relaciones dualistas. [...] Pero del hecho de que esto muestra su carácter negativo o más destructivo para los elementos individuales se concluye, ingenuamente, que la misma función se aplica para la relación del todo (Simmel, 2009: 229-230; traducción propia).

Los ejemplos de los conflictos que contribuyen a la unidad al nivel más alto son, de acuerdo con Simmel, múltiples, variados e interdisciplinarios. Veamos algunos casos. El matrimonio como "formación social" en muchas circunstancias se ve fortalecido no por una uniformidad superflua y engañosa, sino por los "desacuerdos, diferencias internas, discusiones y controversias abiertas entre los cónyuges" (Simmel, 2009: 230). Las animosidades entre las castas en la India no contribuyen a la abolición del sistema, sino todo lo contrario, son "sociológicamente productivas" en el sentido de que mantienen las diferencias y fronteras jerárquicas que están en su base. La competencia entre los individuos en la vida económica juega un papel más bien positivo, aunque pueda ser, al mismo tiempo, "destructiva para las relaciones personales" (Simmel, 2009: 230). La relación erótica consiste en una mezcla del "amor y altivez o la necesidad de dependencia" (Simmel, 2009: 234). La idea de este autor queda clara: el conflicto, juzgado como un fenómeno negativo desde las perspectivas subjetivas e individuales, contribuye de manera objetiva al mejor funcionamiento de un todo más grande.

Pero, iexisten conflictos, guerras y querellas que no contribuyen a un todo más grande? Simmel no lo niega y distingue entre los casos en los cuales el conflicto tiende a ser un terminus ad quem, es decir, un punto de llegada, y los casos en que es un terminus ad quo, un punto de partida. El primer tipo de conflictos conlleva consecuencias desastrosas y destructivas, mientras el segundo contribuye a la formación de entidades orgánicas al nivel más alto.

Aunque el pensamiento de Simmel pueda verse como una mezcla del funcionalismo durkheimiano, psicoanálisis y dialéctica hegeliana (Schermer y Jary, 2013), es una mezcla original y útil para justificar nuestra decisión metodológica: describir los conflictos por herencia, en lugar de los consensos, para arrojar luz sobre las prácticas relacionadas con la repartición de la tierra, con base en el linaje Pasinsa de la comunidad de las Limas, Chenalho'. La hipótesis que se asoma al aceptar la idea de Simmel es la siguiente: los conflictos por la tierra dentro de la familia refuerzan y reproducen las prácticas de herencia en lugar de obrar en su detrimento. Veamos si esta hipótesis es sostenible. 


\section{Relatos sobre los conflictos por la herencia: la voz del primer narrador}

Mi linaje es Pasinsa. Los Pasinsa tenían caballos y mucho terreno. Mi abuelo Antún tenía alrededor de dieciocho hectáreas, era abtel vinik (hombre trabajador). Era también kulej (rico). El kulej posee un terreno apto para el cultivo. Los hombres y mujeres ricos son personas madrugadoras, se levantan temprano para ir a trabajar en el yosil (terreno), porque su mente, su alma y su corazón están enfocados en el cultivo y la producción de bienes de consumo. Son personas habituadas al trabajo arduo; aguantan el calor del sol, el clima frío y la lluvia, son creativas en la planeación y ordenamiento del territorio, siembran frutas, semillas, verduras; nunca les hace falta alimento porque saben hacer producir la tierra para el bien de la familia y la comunidad. El kulej tiene buen corazón; cuando llegan visitantes a su casa, siempre les obsequia algunas cosas, sea frutos, semillas, verduras.

Lo opuesto al kulej es el meon (pobre), al que le falta maíz porque no tiene terreno. El meon a menudo es llamado chabutajel vinik/antzde chabajel, es decir, el hombre o mujer que labra la tierra. Son los jornaleros que no tienen terreno o tienen milpas muy pequeñas y, para sobrevivir, trabajan la tierra de otras personas. Ellos piensan de manera diferente: “¿Para qué voy a buscar terreno si voy a morir? Mejor lo consumo o sólo hago la casa”. Piensan en una casita pequeña. ¿Para qué comprar tierra? Cuando termina su jornada de trabajo reciben 65 pesos por tarea, ${ }^{1}$ se sienten felices. Si trabajan bien, en un día hacen dos tareas y media. Con la ayuda de sus mujeres e hijos ganan más. No invierten en su propia milpa, por eso no pierden nada. En Las Limas, donde hay ochenta familias, seis son chabutajel vinik antz. Yo les respeto, les doy trabajo. Mi hermana Ernestina se casó con un hombre meon. Él es trabajador, sólo que no tiene dinero. Mi hermana tiene cuatro tareas que le regaló mi mamá. Si el hombre meon deja a mi hermana, la tierra le pertenecerá a ella, no a él. No se dice yosil jbol (el terreno de mi cuñado); se dice yosil kixlal (el terreno de mi hermanita). En las familias en las que todos son chabutajel viniketik antzetik se reparte únicamente el solar en partes iguales según el acuerdo de los hijos. Las mujeres en estas familias no heredan nada.

Mi abuelo Antún era kulej, no meon. Les dio terreno a sus hijos Mariano, Sebastián, Manuel, Victorio, Agustín y Miguel. Recibieron los terrenos en Chibtik, que era sikil osil (tierra fría), en Amaltik que era san kixil osil (tierra templada) y en Yutukum y Kanolal que era kixin osil (tierra caliente). Cada familia intentaba poseer tierra en tres climas para tener un balance de alimentación.

Nuestros padres nos ayudaron, crecimos gracias a ellos; ahora ellos son ancianos y nos toca cuidarlos, alimentarlos, curarlos, respetarlos, tal como nos criaron. Esto lo llamamos pak'chi'el (devolver crecimiento) o pak' k'uxlejal (devolver la vida). A cambio, los padres tienen la obligación de donarnos parte de su terreno. En tsotsil decimos spukbeal osil snichnabtak (repartición de la tierra entre los hijos), por eso se dice que la herencia es snaobil stot me li osile (recuerdo del padre y la madre). La herencia es también un símbolo de un recuerdo de utz'alalik (el linaje). A continuación, presento diferentes relatos que versan sobre algunos conflictos en la herencia de la tierra que enfrentamos en mi familia.

\section{Relato 1. La herencia de las mujeres}

Mi abuelo Antún Pasinsa repartió sus terrenos entre sus hijos. A su hija Juana no le dio nada porque antes las mujeres no heredaban la tierra. Había varias razones para ello. Primero, si heredaran las mujeres y se casaran, se duplicarían sus terrenos, lo que causaría inequidades, envidias y celos. Segundo, si el hombre quería a una mujer, tenía que ofrecerle tierras. Si no, se pensaba que no era hombre verdadero, no era abtel vinik (hombre trabajador). Pero había excepciones; por ejemplo, si una familia tenía hijas y no varones les tenía que dar a ellas porque no había de otra. Cuando las hijas se casaban formaban un hogar; si el hombre abandonaba a su mujer, ella regresaba a la casa de su papá. Tercero, las mujeres heredaban otras cosas: la mamá repartía las cosas y el papá, el terreno. "Te doy un gallo o una gallina, unas ollas para nixtamal; si tiene metate, será metate y maíz". Eso le daba su mamá a su hija; a su hijo también le daba un gallo y una gallina para que empezara su 
vida. El papá, aparte de la herencia, le daba también su machete y su azadón.

Mi papá, Victorio, hizo el primer reparto de su terreno en 1978 en Ontik, donde vivíamos, y otro en Mutut. A todos nos dio igual. Daniel, mi jbankil (hermano mayor), tenía entonces quince años, yo trece, Noé diez y Antonio ocho. Cada uno de nosotros recibió diez tareas. Mi papá hizo este primer reparto porque Daniel tenía edad de trabajar la tierra y era ya un hombre responsable. El hijo mayor recibe la herencia para que no necesite trabajar otros terrenos, para que pueda hacer su milpa y sembrar su cafetal, lo que le permitirá buscar una mujer y sostener a su familia en el futuro. Un año después de este reparto Daniel contrajo matrimonio.

El segundo reparto fue en 1998, cuando se dividió parte de veum ka'aetik (potreros) en la curva de la carretera cerca de Las Limas. Este reparto se hizo únicamente entre los hijos que vivían de la tierra. Cada uno de ellos recibió ocho tareas. Las hijas no recibieron nada, y yo tampoco recibí porque no tengo casa allí, no trabajo la tierra. Mis hermanos no me avisaron siquiera de este reparto pues estaba yo en San Cristóbal de Las Casas. Varios meses después dijo mi papá: "Elías no recibió nada, debe recibir algo". En 2001 recibí tres tareas y media en la curva del río como recompensa.

El tercer reparto tuvo lugar en 2008 cuando ya regresé de mi largo viaje. El mismo año, mi papá tuvo el primer tomsbe chichal sjol (derrame cerebral). A pesar de esto todavía podía caminar. En 2011 le dio el segundo derrame; dejó de caminar y trabajar. Se repartió el terreno en los alrededores de Mutut. Esta tierra se llamaba yosilvisente (la tierra de Vicente) porque mi papá se la había comprado a mi tío Vicente Pasinsa. A Daniel le tocó repartir todo en el nombre de papá. Les tocó a todos, aunque los hombres heredaron más terrenos y las mujeres sólo la tercera parte. Las mujeres estaban casadas y no podían tomar decisiones; como no ganaban dinero, tenían que pedir la cooperación a sus maridos, y si ellos no querían cooperar, ellas no tenían el mismo derecho a la tierra que sus hermanos. Se les pedía poca cooperación y por eso asumían poca responsabilidad. Por ejemplo, nosotros, los hombres, cuidábamos al papá cinco o seis veces a la semana y ellas sólo una o dos. Ellas cooperaban menos y los hombres más. Ellas daban treinta o cincuenta pesos y los hombres cien para comprar medicamentos. Y ya que mis dos hermanitas querían más terreno, entonces tenían que participar igual en el cuidado de los padres, lo que era difícil de cumplir. Les dijimos que les íbamos a dar igual en la cuarta herencia: "Está bien, les damos, pero tienen que trabajar esos terrenos, cuidar al papá y cooperar. A ver si cumplen".

La cuarta vez el terreno se repartió cuando mi papá había muerto. Nos tocaba repartir la última parte del potrero que todavía quedaba en Las Limas y otro terreno en el cerro de Chuk'um Ka'atik, que hacía años lo compramos junto con Daniel. Cada uno de nosotros tenía cuatro tareas. En vida, mi papá me pedía este terreno para amarrar su caballo. Luego hicimos un intercambio: en lugar de este terreno me dio ocho tareas en Mutut. Crecían allí tulantik (robles grandes). Mis cuatro tareas fueron divididas entre todos. Esta vez las mujeres querían que se las tomara en cuenta: lask'anik pukbel ko ol yepal li osile (querían que la tierra se repartiera igual). Ernestina argumentó: "Todos éramos responsables de cuidar al papá, de dar cooperación. No sólo a los hombres les debe tocar. La tierra del papá es tierra de todos y, por lo tanto, todos somos iguales". En la curva había otro terreno de mi papá. Cuando todos estábamos reunidos, dijo mi hermana Mari:

Ustedes los hombres recibieron el terreno de nuestro padre. En cambio, a nosotras las mujeres, no nos tocó nada, pero igual cuidamos a nuestro padre y nuestra madre. Igual sufrimos haciendo trabajo para ayudar a nuestros padre y madre cuando estábamos creciendo; dennos este terreno. Dennos, para que nos lo repartamos para nosotras las mujeres. A Elías, nuestro hermano, no le dieron mucho terreno. Él nos acompañará en el reparto del terreno. ${ }^{2}$

Daniel sometió la petición de las mujeres a consideración de todos. Los hombres estaban de acuerdo. Las mujeres cooperaban igual y sus maridos, pues, lo habían aceptado; además ya cuidaban a mi mamá, que era viuda. Hasta ahora se ha repartido todo el terreno salvo 
en Amaltik, el cafetal de mi papá y la casa. En Amaltik se va a repartir igual entre hombres y mujeres porque la cooperación era igual. Mi mamá dice que el cafetal lo quiere Beatriz, quien es la que más la ha cuidado porque vive con ella. Ni ella ni Beatriz quieren que este terreno sea dividido entre todos. Los hermanos sí lo quieren dividir. Daniel dice: "Lo vemos después. A ver quién queda a cuidar a mi mamá". En la última reunión familiar del 21 de julio de 2017, Daniel dijo que Beatriz no podía quedarse con el solar puesto que el rol de cuidar a los padres le tocaba a Benito.

En cambio, en Chibtik, las mujeres empezaron a heredar en la familia de mi tío Manuel, quien al morir su primera esposa buscó a otra mujer, una viuda de Yibeljoj. Su nueva esposa trajo tres hijas a Chibtik. Citaron a los comisariados del pueblo para que testificaran la división de las herencias, y mi tía les dio medidas iguales a todos sus hijos, hijas e hijastros. A estos últimos les dio un terreno de Amaltik, más retirado de Chibtik. Fue entonces cuando los hijos mayores de mi tío se inconformaron porque los otros no eran sus hermanos.

\section{Relato 2. Herencia del solar}

Según la costumbre, el hijo menor debe quedarse, con la familia o sin ella, en la casa de su padre porque tiene la obligación de acompañarlo hasta su muerte. En mi familia le tocaba a mi hermano Benito, pero a él le gustaba estudiar. Encontró trabajo en enfermería en San Cristóbal de Las Casas y actualmente trabaja en el Hospital de Las Madres, donde recibe un salario. Vive ahí con su esposa Yolanda y dos hijos. Le dijimos que él se quedara como heredero, luego lo vio difícil y dijo que no. Quien vive en Las Limas es Beatriz. Ella asume la responsabilidad de cuidar la milpa, ver el cafetal y los caballos, cuidar la tienda, asumir la responsabilidad de la dueña. El dinero de mi papá pasó a Beatriz cuando él se enfermó, y decían mis hermanos: "si se casa Beatriz, si el marido la lleva, ella debe perder el derecho a la herencia. Si el marido llega a vivir en la casa de Beatriz, no la pierde", pues su marido asumirá esta responsabilidad de cuidar a su suegro. Beatriz no se ha casado, no trabaja en el campo, pero tiene dinero para pagar a la gente que trabaje el terreno de su papá, que corte leña. Este dinero viene de la cosecha del café y de la tienda. De eso come y mantiene a su mamá. Ahora está feliz porque adoptó a un niño. Llegó gente a dejarle al niño. "Es mi compañía en mi trabajar", dice. Les pagó tres mil pesos; "tres millones", dice mi mamá. Mis tres cuñadas sentían celos por su hijo adoptivo: "¿Por quéle dan herencia?", preguntaban. Dijo Beatriz: "Vu'un tajnop jtuk" (eso lo pensaré yo sola). De nombres y apellidos le puso Edgar Melquisedec Pérez Pérez, como si fuera su hermanito. Melquisedec era un sacerdote que no tenía linaje, ahora Edgar ya lo tiene. Por mucho tiempo se pensaba que Beatriz se haría la dueña de la casa al morir mi mamá, pero en la reunión familiar del 21 de julio de 2017 Daniel argumentó que Beatriz, como mujer, no se podía encargar del solar y le tocó a Benito. Pienso que esto es injusto porque él no cuidaba de mi papá, vive en San Cristóbal y no va a cumplir con sus deberes. Creo que en el futuro será Beatriz quien se quedará con la casa y el solar. Ella aceptó la decisión de la familia, sólo dijo: "Oyepal jch'obtik, chiamtej" (tengo mucha milpa, trabajo) Con eso quiso decir que es igual al hombre o abtel vinik, es decir, que es hombre-mujer.

\section{Relato 3. Venta ilícita del terreno a otro linaje}

En febrero de 2017 a Antonio le tocó su herencia en las Limas, del río hacia la carretera. Era un espacio bonito porque allí, gracias a la cercanía de la carretera, se podía construir una casa o instalar una tienda. Pero Antonio necesitaba dinero y decidió vender a otra persona que no pertenece al linaje. Daniel supo que en ese terreno estaban almacenados bloques de cemento y le preguntó a la esposa de Antonio si eran de ellos. Ella respondió que no, que eran de otra persona. Así comenzó la sospecha y me dijo Daniel: "Pregúntale a Antonio si vendió su herencia". En aquel tiempo Antonio estaba trabajando en construcción en Puerto Vallarta. Yo le pregunté si había vendido su terreno y me respondió que no. Después de algunos meses regresó Antonio a las Limas. En marzo de 2017 Daniel nos reunió a toda la familia en la casa de mi mamá y le preguntó de nuevo a Antonio si había vendido el 
pedazo de terreno, a lo que él respondió: "No lo he vendido. Sólo quedamos en un acuerdo de hacer un cambio de terrenos. Yo le voy a dar el terreno de la carretera de pedregal y él a cambio me va a dar un terreno plano cerca del río para sembrar milpa y frijol". "iEntonces has recibido dinero? Dime la verdad". Dijo: "Si”. "¿Por qué?”. Y dijo Antonio: "Porque tuve necesidad de dinero. Tengo derecho de intercambiar o vender porque es mi herencia, no es el terreno de ustedes". Daniel respondió: "Es el terreno de papá, hay un croquis". Sacó el croquis y se lo mostró: "Éste es el terreno total que tenía el papá. Este terreno queda para los hijos. No vamos a permitir que otro linaje entre en el terreno de la familia". Y respondió Antonio: "Tú, hermano, te pones muy duro contra mí. Tu hijo Efraín vendió el terreno en Tenejaltik, que es la herencia que te había dado el papá. ¿Cómo es posible que no permitas que yo venda mi herencia?". Daniel respondió: "Ese terreno no tiene croquis, por eso se lo di a mi hijo como herencia. Estoy defendiendo el terreno cerca de la carretera porque tienes hijos. Probablemente en el futuro, cuando crezcan, lleguen a tener dinero, puedan construir casas y hacer sus tiendas, porque los terrenos a la orilla de la carretera son muy atractivos. Si lo vendes a otro linaje, tus hijos sólo quedarán mirando que serán los del otro linaje quienes construirán allí sus casas. Si vas a vender y el que compre quiere sacar su escritura, no la voy a firmar ni autorizar. Se quedará sin escritura". A lo que Antonio respondió: "Lo voy a pensar".

Daniel tiene el croquis y los documentos de compraventa, y sin eso no es posible obtener escritura. El hermano mayor tiene el derecho de guardarlos en nombre del papá. Si Daniel muere, estos documentos quedarían en mi resguardo porque soy el segundo hijo.

Hay más casos en la historia del linaje sobre personas que vendieron su herencia a otros linajes. Moisés, el primer hijo de Agustín, vendió sus terrenos en los años sesenta, tal vez por la muerte de su mamá y su papá y por la política, a la cual se dedicaba bajo la influencia de su papá en Chenalho’ y San Cristóbal de Las Casas. Moisés no supo apreciar a su abuelo ni a su padre porque vendió parte de la herencia y prefirió borrar el recuerdo en su mente y su corazón; prefirió comprar terreno de otras familias. Quizás stub’ ta sjol ta yo ‘ontonk'u usitik akbil komel yu 'un li stot sme 'e (borró de su cabeza y de su corazón lo que le quedaba de su padre y madre). Su terreno en Yutukum lo vendió a Eliseo, su primo. Vendió una hectárea o más en Chibtik y vendió en Amaltik. Es una persona desarraigada; ahora está vendiendo un pedacito en Chibtik y dice, pues, que no lo quieren porque había apoyado a Rosa Pérez Pérez, la alcaldesa de Chenalho'. ${ }^{3}$ Dice que quiere olvidarse de la comunidad. Él sólo avisó a quien quiso, así corrió la voz. Si uno dice: "Esto está muy caro, va al otro". No tenemos derecho de vender la herencia porque vender indica que no apreciamos a nuestros padres, se borra el recuerdo. Me daría vergüenza decirles a mis hijos: "Eso me dio mi abuelito, pero lo vendí". Normalmente es el hermano mayor quien decide si el terreno del papá se puede vender o no. Es él quien guarda el croquis. Moisés vendió el terreno sin croquis porque era su herencia, pero incluso si tuviera el croquis, también lo podría hacer porque es el hermano mayor. No hay otro control. Si él como hermano mayor empieza a vender la herencia, los demás también venden y así se acaba la herencia.

\section{Relato 4. La venta ilícita del terreno dentro de la familia}

Mi mamá vendió a Víctor un pedazo de terreno que ella iba a compartir con Beatriz y Benito. Mi mamá habló con cada uno, pero no nos reunió a todos. Daniel no sabía nada y platicó con nuestra mamá: "Está mal lo que hiciste, hubieras consultado con tus hijos; cualquiera que quiere vender debe consultar a los demás". Nuestra madre no respetó la regla. Decía que necesitaba dinero para su medicina, pero era mentira porque todos cooperamos para comprársela. "Y quiero dar a la iglesia”, dijo también. Además mencionó que había sufrido con mi papá: "Este terreno es mi parte". Cuando mi hermano le reclamó, mi mamá lloró: "¿Por qué no puedo vender el terreno que es mío?, tengo derecho a vender". Víctor pagó por ese terreno cinco mil pesos. En realidad, Daniel no le reprochaba a mi mamá que hubiera vendido su terreno a Víctor, sino que lo hiciera a escondidas. 
Podemos tomar decisiones, pero debemos avisar a todos. Por ejemplo, yo vendí mi terreno de seis metros por cincuenta a Ernestina. "Lo quiero, tengo dinero", dijo mi hermanita. El dinero se lo dio Rosa, la alcaldesa, para invertir en una obra importante para la comunidad. Alux, el marido de Ernestina, compró una motosierra. Más tarde dijo Ernestina: "Tengo cuatro mil, véndeme otro". Le contesté: "El terreno que tengo en Chibtik vale ocho mil". "No tengo tanto", dijo. Tampoco me pagaron por aquel terreno que les vendí. Dijo Alux: "Mejor te voy a limpiar tu cafetal y así te pago el terreno". No sé si Alux limpió mí terreno. Se lo vendí tan barato por mi edad, porque no puedo trabajar tanto terreno y porque cuando no tengo a quien dejar partes pequeñas yo me muera. Hay otras razones: Ernestina cuidaba mi turno para acompañar a mi mamá: tajchin jmekutik patbe kutik yo'onton (le acompañamos para que no se sienta sola). Cuidar a mamá significa dormir allí en la noche y convivir con ella en el día. Entramos a las seis de la tarde y salimos a las seis del otro día para que no se sienta sola. Ernestina llegaba entonces como mi sustituta, hacía la comida, llevaba su frijol, su maíz, allí torteaba, molía su maíz, comía con mi mamá. Yo le llevaba cosas, platos, ollas. Cuando yo llego, Ernestina está allí y me ayuda a cocinar. Los hermanos lo aceptan porque saben que vengo solo, que no me acompaña mi mujer. Y Ernestina es mi kox (hermana menor) y le toca, y también porque vive cerca.

Entonces, aquella vez en la reunión encabezada por Daniel se acordó que Víctor debería dar mil pesos más para mi mamá y una reja de refrescos por no respetar a sus hermanos. Antes se hizo una propuesta: "Te damos una mitad y otra mitad a Benito". Víctor no aceptó. Cuando aceptó dar mil pesos más, todos estuvieron de acuerdo. Fuimos a poner tz'ak en ese terreno. Había que encontrar la stuk'il osil (línea perpendicular). Daniel se paró, lo checó con la vista, llamó a Noé y le pidió que también lo checara. Indicaron el punto donde iban a poner el tz'ak. Llamaron a Víctor: "Abre hoyo y siembra el tz'ak aquí". Víctor bajó, abrió un hoyo y puso el tz’ak. Él quería poner el mojón lo más lejos posible para que fuera a su provecho. "Hasta aquí es la dirección derecha”, le corrigió Daniel. Su mujer protestó, pero Víctor tenía que obedecer.

\section{Relato 5. Conflicto por la colindancia}

En 2016, después de la muerte de mi papá, tuve un conflicto con Ernestina en Mutut. Ella sembró sus matas de café en la primera colindancia y no respetó la regla de dejar una brazada de distancia - en caso de milpa es un metro- Cuando fui a ver mi terreno me di cuenta de ello, pasé a ver la colindancia y observé que su café estaba sembrado en la orilla. Fui a Las Limas y le pregunté por qué no respetaba las reglas. Le dije que tenía que arrancarlo: "Si no lo haces, yo lo voy a arrancar". Pasaron muchos meses; en junio de 2017 fui a ver y ya lo había arrancado. Obedeció. Si no hubiera obedecido, yo lo arrancaría, y si yo lo arrancara, lo dejaría tirado y le avisaría para que tuviera tiempo de trasplantarlo. Hay que respetar los acuerdos, porque si no se respetaran de buena manera, me sería permitido arrancar su café, pero esto no es muy conveniente porque, entonces, yo y mi cuñado quedaríamos como enemigos. Hay una segunda opción: podríamos acudir a Daniel para que él vaya y vea dónde están sembradas las matas del cafetal, y él, como jbankil, podría intervenir y decir a mi cuñado que arranque su café. Esto tampoco conviene porque crea enemistades entre Daniel, mi cuñado y yo. La tercera opción es la más viable: Daniel puede convocar a los integrantes de la familia para atestiguar y buscar la solución. Toda la familia decide: "Lo arrancas o lo arrancamos". Ya no puede decir mi cuñado que fue Daniel o fui yo. Para no buscar enemistades hay que decidir entre todos. Para mantener unida la familia se debe fomentar la comunicación permanente en cualquier decisión.

\section{Relato 6. Servilismo como estrategia para apropiarse del terreno}

Víctor es mi hermano sexto, mi kitz'in. Tiene seis hijos. Había veces que él deseaba quedarse con la casa de mi papá. Lo que hacía era que llevaba la comida a mi papá y a mi mamá de todo lo que cosechaba. Sobre una persona así decimos en tsotsil k'uxtayo'onton stot sme (es servicial con su papá y mamá). A cambio de sus servicios, les pedía terreno: "Préstame este terreno, voy a sembrar 
milpa”. "Aquí está", decía mi papá. Y al otro año: "Voy a sembrar chícharo". "Bueno, está bien, hijo". Luego café: "Está bien, siembra tu café". Luego les dice: "Ya coseché la milpa, tenemos elote; ya coseché chícharo, vamos a comer. Ya coseché mi café, vamos a tomar café". Luego le decía a mi papá: "Dame este árbol para mi leña y tu leña”. "Ahí está”. Mi mamá decía: "Es el único hijo que me atiende bien".

Cuando llegó el tiempo de repartir la herencia en Mutut entre todos los hijos, mi hermano Daniel dijo: "Vamos a repartir el cafetal de mi papá". Eran tres o cuatro tareas de cafetal. Víctor comentó: "Éste es el terreno que me dio mi papá; por eso no se puede repartir". Pero mi hermana Beatriz lo negó: "La xch'amun no'ox, ali jtote muyuk bu lajyak'be o"' (él sólo lo pidió prestado, mi papá no se lo dio para siempre). Preguntamos a mi papá si era cierto y confirmó lo que decía Víctor, que se lo había dado para siempre, pero nosotros contestamos: “¿Cuándo se lo dio? No lo sabemos. Este cafetal queda para nuestro papá, no para ti". Víctor se enojó con nosotros, pero hicimos lo correcto porque nadie puede tener más terreno que otros, nadie puede vender o comprar a escondidas sin que toda la familia esté presente.

\section{Relato 7. Apropiación ilícita del terreno}

Yo también pedí el terreno a mi papá porque me habían sacado de la lista. Decían mis hermanos: "Elías es maestro, no necesita terreno para comer". Entonces le pedí tierra a mi papá y me dijo: "Este terreno es mío, trabájalo". Ese mismo año pedí a mi cuñado Antonio Taquimut que me vendiera hijuelos de plátano y que los sembrara alrededor. Antonio ha sido parte de la familia desde 2003; luego murió su esposa, mi hermana Ester y él se quedó con las tres hijas en la casa que está pegada a la de mi mamá. Aunque se casó con otra mujer hace tres años, lo seguimos tratando como familia. Víctor, mi hermano menor, trabajaba como su chalán. Cuando yo me fui a España a estudiar el doctorado dos años más tarde, Víctor, que ya estaba casado, me pidió permiso para xchaman kosil (arrendar mi terreno), y ahí sembró chícharo, milpa y frijol. Cuando regresé de España le dije: "Ya no vas a trabajar mi terreno". Él me contestó: "No, es mi terreno, nosotros con mi esposa sembramos el plátano". No me lo quería devolver; se apropió de mi terreno. Dijo que lo había trabajado por quince años y que en la Ley Agraria dice que, si alguien trabaja siete años, el terreno es suyo. Le mencioné a Daniel: "Víctor es un mentiroso. Dice que sembró plátano con su mujer pero es mentira, pues entonces no tenía mujer". Daniel le dijo que no eran quince años, sino apenas cinco, y Víctor le contestó: "La Ley Agraria me protege". "Tu ley no vale”, respondió Daniel. Víctor le contestó: "iVoy con el comisariado!", y Daniel le respondió: "El comisariado no manda en esta familia. Nosotros mandamos y el comisariado tiene que obedecer. Si decimos que es de Elías, es de Elías; ni el comisariado puede quitarlo". Luego Daniel me instruyó: "Paga a gente, trabaja el terreno. Y tú, Víctor, ya no toques ese terreno".

\section{Relato 8. Reparto de los árboles}

El día 27 de febrero de 2017 había dos tareas que desarrollar; repartir los cipreses y el terreno. Salimos a las 6.30 de la casa. En cuanto al reparto de árboles, empezamos a contar los cipreses, que son de mucha importancia porque sirven para hacer tablas, vigas y reglas. Mi mamá a la fuerza quiso ir con nosotros: “¿Qué tal si hay problemas? iRepartan igual!". Cuando llegamos a Mutut había dos propuestas. La primera era contar los árboles juntos, y la segunda dividirnos en dos grupos: uno empezando por el lado de lok'em k'ak'al (salida del sol, este) y otro por el olon maleb k'ak'al (poniente norte). Ganó la segunda propuesta porque era más rápida, más ágil. Yo fui con Manuel y con Antonio Taquimut. En el otro grupo, que fue a olon maleb k'ak'al, estaban Daniel, Víctor, Benito, Agustín, Beatriz y la mujer de Antonio, María. Nosotros éramos menos porque íbamos a correr mucho, el camino era muy largo. En total había más o menos 133 cipreses. Se discutió cómo se iban a repartir y mi mamá también pidió algunos: "Se están pudriendo las vigas de mi casa". Unos dijeron: "Vamos a contar los arboles más grandes". Otros dijeron: "Vamos a recorrer once árboles cada uno para saber cómo repartirlos entre todos", iniciando con Ernestina porque allí estaba su 
pedazo de terreno. Después seguí yo porque mi terreno quedaba cerca, después Taquimut, después Mari, después Víctor, después mi mamá, después Beatriz, después Daniel y después la esposa de Antonio, que se encontraba allí en su nombre. Todos estábamos tranquilos y nos habíamos reunido para agradecer a Dios. Algunos árboles sobraron y seleccionamos ocho grandes para la iglesia. Entonces dijo Víctor: "A mí me tocaron muy chiquitos". Y le siguió Manuel, el esposo de Ernestina: "También a mí". A Manuel le recompensó Daniel porque sus árboles realmente estaban chicos y se le dio un árbol más. A Víctor le tocaron nueve árboles porque antes había cortado tres sin aviso, que los tenía almacenados en la cocina de mi padre. Víctor xlaj yu'un te' (tiene ansia de poseer más madera). Él es ch'oj te' (carpintero), fabrica sillas y mesas, y las vende. Decimos: Víctor sa'k'op (busca pleito), es ch'opol sjol (mal de cabeza). Beatriz tenía la llave de la cocina, pero él metió la madera por un hoyo. Yo llevaba otra llave, abrí en presencia de mis hermanos y nos dimos cuenta de que allí estaban. Daniel dijo que Víctor no respetaba a la familia. Todos dijimos que se repartiera su madera, pero Víctor protestó, dijo que le había costado dinero cortarla. Por abusivo seleccionó los árboles grandes. Dijo: "Mejor me den los nueve, y los tres que los quiten de la cuenta”. Así hicimos. Todo quedó calmado.

\section{Relato 9. El cuñado es parte de la familia}

Mi hermanita Ester nació el 18 de marzo de 1968 en Chibtik. A los 24 años de edad quiso contraer matrimonio con el joven Antonio Pérez Taquimut, que pertenecía a la religión tradicional maya. Ante esa diferencia de creencias, mi padre se opuso. Taquimut acostumbraba ir a las fiestas del pueblo y regresaba ebrio de alcohol de caña. Victorio consideraba al joven Antonio Pérez Taquimut como una persona que adoraba a Satanás y le hacía obras. Ester lloraba. Con el tiempo, los hermanos y los padres autorizaron el matrimonio y Ester se fue a vivir con su marido a la comunidad de Vayemvakax, cerca de Yambteclum, donde procrearon cinco hijos: cuatro niñas y un niño. Las diferencias religiosas terminaban en pleitos constantemente. Cuando mis padres insistían en que ella asistiera a los cultos, el marido se incomodaba y resolvía el problema emborrachándose, insultando y maltratando a su esposa. En 2002 Ester se inscribió en el Programa Oportunidades, mediante el cual el gobierno mexicano otorgaba apoyo a los titulares de las familias, y se inscribió en el programa de operación de trompas de Falopio. Después de la cirugía empezó a sufrir efectos secundarios. Fue tratada por los paramédicos presbiterianos, por curanderos tradicionalistas y por doctores alópatas en Tuxtla y en San Cristóbal de Las Casas. Los diagnósticos diferían. Los médicos alópatas diagnosticaron osteomielitis y artropatía secundaria, los curanderos brujería, y los presbiterianos delimitaron la causa en los pecados de Antonio. Fue entonces cuando éste y su esposa se mudaron a la casa de mis papás, y mi mamá le donó parte del solar donde Taquimut construiría después una casa. El esposo de Ester comenzó a entristecerse porque veía que su esposa no mejoraba, y ella decía: "Mi esposo está muy triste y llora mucho, y mis cinco hijos. Tengo que vender el terreno. Mi esposo me dice: 'Te voy a seguir curando hasta que salgas de tu enfermedad"'. La venta de bienes, de 15000 matas de café y de dos hectáreas de terreno resultó ineficaz porque no logró sanar su mujer. Los hermanos y padres de Ester comenzamos a presionar al esposo, y Daniel dijo lo siguiente: "Te decíamos que era culpa tuya que no sanara tu mujer. Nuestro Dios no capta ni escucha nuestra petición. Si aceptas ser bautizado dentro de la religión, serás salvado, así también se salvarán tu mujer y tus hijos. Si vuelves a beber cuando estás bautizado será una perdición para siempre y no alcanzarás el perdón de tus pecados por parte de nuestro Dios". Mi hermanita Ester murió en diciembre de 2004. Antonio Taquimut sigue viviendo en la casa al lado de la de mi padre cuidando a los hijos. En 2016 contrajo nuevamente matrimonio con una mujer tradicionalista.

Antonio Taquimut tenía derecho a heredar la tierra de nuestro padre como todos nosotros pues representaba a mi hermanita Ester. Recibió otro solar en Cipreses, que era Mutut todavía cuando vivía mi padre. Después de su muerte, le tocó en Veum Ka'tik, en la curva, y un pedazo en La Cruz, en el Cerrito. Heredó 
terrenos hasta el último reparto en mayo de 2017 porque dejó de cooperar con dinero para cuidar a mi mamá. Lo hizo probablemente porque ya sabía que el único terreno que nos tocaba era Amaltik, un lugar muy lejano que no le convenía heredar. Es por eso que se separó del grupo. Dijo: "Mu xilaj yu'un jutem osil, oy ep banamil" (no me muero por un pedazo de tierra, tengo bastante). Entonces mis hermanos respondieron: "Nosotros no despreciamos ni apartamos. Se ve bonito, estamos unidos como familia. Porque tú tienes tus hijas e hijo que son nuestros sobrinos. Aunque Ester tu esposa esté muerta, para nosotros vive. Tiene sus hijos e hijas aquí. Que el terreno quede como recuerdo de su madre y de su abuelo". Pero Taquimut no ha cambiado su decisión.

\section{Las reglas y las estrategias: la voz del segundo narrador}

El texto anterior es de autoría de Elías Pérez Pérez, hijo de Victorio, el líder principal de los cambios ocurridos en los años cincuenta en Chibtik, un paraje de Chenalho'. La vida y obra de Elías Pérez Pérez ocupa un lugar especial en la historia de su linaje, en Chenalho', e incluso en todo el Chiapas indígena. Su odisea desde sus estudios de primaria en Xunuch, Chibtik, Chenalho' y Chojolvo', y de secundaria en Chenalho', hasta sus estudios superiores en la Universidad Politécnica Nacional y en Salamanca, España, fue descrita con detalle en otro texto (Jacorzynski, 2008).

Durante mis estancias en Chibtik en 2013 y 2017 aprendí muchas lecciones: una de ellas fue que el motivo de pleitos, odios, celos y problemas, o lo que en batz'í k'op llaman k'opetik (palabras, riñas), se encontraba en conflictos familiares por la herencia. En 2017 pedí a Elías que relatara algunos conflictos relacionados con este tema en Las Limas, comunidad a la que sus padres se mudaron en los años ochenta, y en esta parte comentaré sus relatos.

Antes de hacerlo es menester mencionar algunas nociones que abrirán el camino al análisis. Dos ideas iluminan este ensayo. La primera es una afirmación general: la vida social se puede describir como un conglomerado de prácticas que se vinculan en diferentes relaciones descriptibles dentro de una forma de vida. La segunda idea consiste en que, a partir de la descripción, reconstruiré las estrategias y reglas correspondientes a las prácticas.

En primer lugar, intentaré resumir los relatos de Elías según los conceptos expuestos en la introducción. ¿Qué es lo que realmente se practica en Las Limas cuando se habla sobre herencia? En tsotsil se dice chich spukel k'usitik oy yu'un li totil meil (recibir cualquier cosa del padre o la madre) o snaobil stot sme (el recuerdo del padre y la madre). En estas expresiones generales se ubican diferentes subprácticas como las siguientes: el reparto de gallinas, gallos u ollas en el caso de las mujeres, y de azadones, hachas y machetes en el caso de los hombres para su slikebal skuxlejal (inicio de la nueva vida); ja' li bankilale ja' to 'Tasbis li banamile (el hermano mayor efectúa la medición del terreno); chich' tz'unel tz'ak (poner mojones para marcar el terreno); chich' chakel sat te'etik (reparto de frutas); chich' pukel yosil (reparto del terreno) y tzomsba utz'alal chapanik k'op (reuniones entre familia para arreglar los problemas) por el hermano mayor, quien desempeña el papel de tzobsvanej ta utz'alal (el convocador de las reuniones de la familia), para manel-chonel osil (compra-venta del terreno).

¿En qué circunstancias, cuándo y dónde se efectúan las prácticas de herencia? En este caso destacan dos espacios: el primero es la casa de Victorio antes y después de su muerte. Allí se reunían todos los participantes/herederos para desayunar, comer o cenar, cuidar a los padres y resolver problemas. En la sala se sentaban a la mesa los hermanos y hermanas para platicar, expresar sus puntos de vista, compartir sus dudas, acusar o planear los repartos. Las reuniones y convivencias se organizaban antes y después de las salidas destinadas a medir los terrenos; a partir de la muerte de Victorio las convocó por el hermano mayor, Daniel, que solía reunir a toda la familia en la casa de la madre para buscar soluciones a los conflictos (relatos 3 y 5). Es también la casa el símbolo del manejo y del control; es allí donde se guarda el croquis de los terrenos comprados, que se hereda del padre según el criterio de la edad: "Si Daniel muere, este documento quedaría en mi resguardo porque soy el segundo hijo" (relato 3). 
El segundo espacio donde se efectúa la mayoría de las prácticas de herencia es el campo, el lugar del reparto. Las prácticas más importantes realizadas a la intemperie son: 1) el chequeo de la colindancia: "Cuando fui a ver mi terreno me di cuenta [...]" (relato 5); 2) la medición de la colindancia: "Fuimos a poner $t z$ 'ak en este terreno [...]" (relato 4), y 3) el conteo y reparto de árboles: "En total había más o menos 133 cipreses" (relato 8).

En cuanto a las personas que participan en las actividades relacionadas con la herencia, se analizarán bajo dos aspectos: el rol social/parentesco y el género.

Elías tenía nueve hermanos y hermanas, y entre el grupo de herederos se encontraba también Antonio Taquimut, el esposo de una hermana fallecida, Ester, a causa de una cirugía mal hecha y la negligencia de los médicos alópatas (relato 9). La razón principal de la inclusión de Antonio era el hecho de que "representaba" a Ester. El derecho a esta representación residía en que cuidaba de los cinco hijos de él y su difunta esposa, además de que vivía en el solar de su suegro. Esta situación se mantuvo hasta julio de 2017, cuando Antonio se autoexcluyó del último reparto. El otro sbolo cuñado de Elías era un meon, pobre. Como chabutajel vinik (hombre labrador), no tenía tierra ni tendría derecho al terreno de su esposa si se separara de ella.

La participación en las prácticas de herencia está circunscrita en especial a los hijos e hijas, aunque también intervienen los primos y tíos si se toman en cuenta otras prácticas relacionadas, como la compraventa de terreno (relato 3), porque quienes intervienen en la herencia, en la mayoría de los casos, son miembros del mismo linaje, aunque no necesariamente de la misma familia. Al grupo de herederos futuros pertenecen también los niños adoptados por los miembros como el hijo de Beatriz, Melquisedec, cuyo derecho a la tierra del padre de Elías es cuestionado por una de las hermanas de este último; sin embargo, Beatriz defendió a su hijo: "Eso lo pensaré yo sola" (relato 3). Edgar Melquisedec "permanece" beneficiario, a partir de su adopción, de la herencia que le dejará Beatriz, su madre adoptiva. Finalmente, en cuanto al género, heredan tanto los hombres como las mujeres, aunque esta costumbre es relativamente reciente (relatos 1 y 2 ). Cabe resaltar otro hecho: las prácticas de herencia descritas por Elías se circunscriben a las familias que poseen tierra, pero no pueden presentarse como una tradición homogénea "indígena" de Chenalho': en las familias de los chabutajel viniketik antzetik, el objeto de la herencia entre los hermanos varones es sólo el solar. De esta manera, los jornaleros no caben en el cuadro pintado por Elías, en el cual el motivo principal de la actuación es la posesión de la tierra, porque los jornaleros "piensan de manera diferente".

A modo de completar la imagen de quienes participan en la herencia vale la pena mencionar sus intereses, estrategias, motivos y estilos. Los conflictos relatados por Elías revelan cuatro características generales. En primer lugar, una motivación recurrente es el deseo de recibir el terreno de más calidad - por ejemplo, cafetales-y de extensión más grande posible. En segundo lugar, las estrategias tomadas por los actores son racionales en el sentido de Barth: tienden a maximizar el beneficio de su propia familia nuclear en contra de los intereses de los hermanos y hermanas (Barth, 1981: 38). En este sentido, Elías considera que Antonio Taquimut decidió autoexcluirse de los últimos repartos porque el terreno que le tocaría en Amaltik es un "lugar muy lejano que no le conviene heredar" (relato 9). En tercer lugar, la racionalidad tiene también otro rostro: todos deben abstenerse de sembrar discordia entre la familia — así por lo menos piensa Elías, quien alude a diferentes estrategias a la hora de resolver el conflicto con Ernestina (relato 5) - . En cuarto lugar, las estrategias no siempre son individualistas. En el primer relato, las mujeres persiguen un interés grupal y forman una coalición, en la que incluyen también a Elías, en contra de los demás hermanos: "A Elías, nuestro hermano mayor, tampoco le tocó bastante terreno" (relato 1). La forma kutik utilizada por Mari es un plural excluyente. Ella habla no en nombre de todos los hermanos, sino de las mujeres y de Elías, en contraposición a los demás hermanos, que representan el grupo de los contendientes.

Los otros conflictos tienen carácter individual. En el relato 5 Elías se encuentra en una contienda con Ernestina - es el típico conflicto por colindancias-y 
en el relato 7 con Víctor: "No me lo quería devolver; se apropió de mi terreno". En ambos casos los contendientes recurren a las reglas de herencia, que no son suficientes para resolver el conflicto. También recurren a Daniel, el hermano mayor, quien tiene autoridad para convocar una reunión y dar la razón a uno de los contendientes. En cuarto lugar, en los demás relatos el conflicto se produce entre uno en contra de los demás hermanos. Éste es el caso de Antonio, quien planea vender su herencia a otro linaje (relato 3), y también de Víctor, quien compra el terreno de su mamá a escondidas (relato 4), se vuelve servil para apropiarse del terreno de su papá (relato 6) o exige más árboles (relato 8). En estos casos es el hermano mayor, Daniel, quien habla en nombre de todos.

Elías acepta que es lícito desear la herencia y competir por ella; él mismo pidió más terreno a su papá cuando se sintió excluido de un reparto (relato 7), por lo que entiende las razones de sus hermanos cuando lo quisieron a su vez excluir. Él tiene más recursos económicos, vive en San Cristóbal, es maestro y no depende de la tierra, pero eso no le impide desear la herencia como los demás y luchar por ella en el escenario de la competencia familiar. Lo que no acepta es la conducta de Víctor, su yitzín, porque utiliza el servilismo y la lisonja, miente (relato 7) y muestra codicia por la madera (relato 8). A esto se une su deseo de saltarse las reglas acordadas en la familia y buscar justicia en instituciones externas como el comisariado y la Ley Agraria, lo que lo pone en ridículo a los ojos de Daniel, quien lo exhorta de manera decisiva: "El comisariado no manda en esta familia” (relato 7). Víctor es para Elías una oveja negra porque utiliza cualquier medio para satisfacer su codicia. Víctor sa'k'o (busca pleito) y tiene chopol sjol (mal de cabeza).

¿Qué estrategias utiliza Elías? Sobre esto habla en el relato 5, donde menciona tres opciones para resolver el conflicto con Ernestina: en primer lugar, puede arrancar el caféél mismo, pues ella violó las reglas de colindancia; como segunda estrategia, puede acudir a Daniel para que éste intervenga con el marido de Ernestina y le exija que arranque el café. Estas dos estrategias, aunque estén permitidas, no son las mejores para Elías porque “crean enemistades" entre él, Daniel y Miguel, el esposo de Ernestina. En cambio, la tercera estrategia, que consiste en convocar una reunión de toda la familia, es la preferible: "Llega la familia y decide: "lo arrancas o lo arrancamos". El ideal de Elías es "mantener unida la familia" a través de "la comunicación permanente".

A continuación se reconstruyen algunas reglas generales que se siguen en el reparto de la herencia, las cuales pueden ordenarse de la siguiente manera:

1) La tierra y otros bienes sometidos a herencia deben dividirse equitativamente entre los herederos definidos como hermanos y hermanas o sus representantes -hijos adoptivos e hijos de los hermanos fallecidos(relatos 2 y 9). Esta regla puede caracterizarse como el "principio de equidad" que, paradójicamente, no siempre contribuye a una mayor igualdad dentro de la familia porque, aunque las nuevas condiciones de vida, sobre todo la migración de algunos miembros de la familia a las ciudades y sus ausencias largas (relato 7) puedan obstaculizar su acceso a la herencia, esto no los excluye del reparto de bienes. Si las personas con recursos desiguales dividen de manera igual, el resultado no es mayor igualdad, sino más desigualdad. En este sentido, las relaciones de desigualdad dentro de la familia toman formas diferentes, siendo la más común la práctica de que los miembros más ricos den empleo a los más pobres.

2) La tierra y otros bienes sometidos a herencia deben dividirse entre los hijos de manera proporcional a los servicios que brindan a los padres. Esta regla se puede expresar como el "principio de reciprocidad" (relatos 1, 2 y 9). De esta regla general se desprenden varias específicas: por ejemplo: 2.1) la tierra debe pertenecer a quien la cultiva y la cuida (relato 7); 2.2) el primer heredero es el hermano mayor porque ayuda al padre en el trabajo de campo; 2.3) el hermano menor tiene la obligación de quedarse en la casa de sus padres para ayudarles cuando envejecen por lo que recibe, a cambio, el solar (relato 2).

3) Las decisiones deben tomarse, preferiblemente, entre todos los familiares sin recurrir a instituciones externas (relato 4). Esta regla puede interpretarse como el "principio de autonomía familiar". 
4) Se deben respetar las decisiones tomadas anteriormente por los miembros de la familia. Esta regla se observa en el conflicto por la colindancia (relato 5) o en dudas acerca de la transparencia de las transacciones intrafamiliares (relato 4). Se puede identificar como el "principio de consecuencia".

5) Finalmente, la tierra heredada debe conservarse en el mismo linaje o en la familia extensa patrilineal y se prohíbe la venta a los miembros de otros linajes. La venta sólo se permite a los descendientes paternos del linaje (relato 3). Esta regla puede denominarse el "principio de la solidaridad del linaje".

Estas reglas, que se desprenden de la descripción de los conflictos elaborada por Elías, muestran una característica interesante: las posibles contradicciones entre los principios. Veamos dos ejemplos. El principio de equidad puede entrar en conflicto con el principio de reciprocidad. El hermano menor hereda el solar porque es él quien vive con los padres y los cuida, mientras que la razón por la cual el hermano mayor tiene más privilegios en los primeros repartos es que es él quien más ayuda al padre. Asimismo, quien cultiva la tierra tiene más derecho a ella que quien la deja en baldío. En otras palabras: en la familia de Elías todos son iguales, pero algunos son más iguales que otros por sus méritos. Otro ejemplo es el siguiente: el principio de equidad puede entrar en conflicto con el principio de consecuencia. En este sentido, las decisiones que tomaban el padre y la madre de Elías favorecían a Víctor, hecho por el cual fueron criticadas con base en el principio de equidad. ¿Cómo resolver un conflicto en el cual una parte alude al principio de equidad y otra al principio de consecuencia?

La solución aceptada en la familia de Elías se deja expresar como una metarregla a la cual se alude en caso de conflicto, a saber:

6) En caso de conflicto entre las reglas mencionadas, quien debe encargarse de interpretarlas o resolver las contiendas es el representante de la familia: el padre o, en su ausencia, el hijo mayor (relatos 1 al 9). Esta regla puede denominarse como "metaprincipio pragmático-patriarcal”, designación opaca y artificial pero que resume bien el contenido. Es pragmática porque se recurre al juicio de una sola persona que decide sobre un caso dudoso, lo que ahorra tiempo a los demás hermanos, y es patriarcal porque esa persona es hombre, no mujer. Obsérvese que en los casos de crisis es Daniel quien tiene la última palabra; es él quien toma las decisiones, quien guarda el croquis, escucha, invoca, enseña, corrige, revoca y amonesta. El poder que tiene Daniel es a primera vista autoritario, lo que se observa en que tanto Antonio como Víctor se enojan con él por las decisiones que impone. Pero este autoritarismo es moderado porque él no representa sus propios intereses, sino los de la familia, de modo que, en última instancia, es la familia la que decide, no Daniel el que impone. Considero que así deben entenderse las palabras de Elías sobre "mantener unida la familia" a través de "la comunicación permanente". Comprendemos ahora mejor, dicho sea de paso, la idea de Simmel relacionada con que, aunque los conflictos siembren discordia y atormenten la vida familiar, son indispensables para el acto de autopoiesis de las prácticas y el desarrollo de la personalidad de los actores. (Simmel, 2009: 305) los conflictos enseñan qué reglas se han evadido, imponen la obligación de juntarse y compartir la palabra y los espacios, y hacen recordar a todos que la autoridad paterna y la del hermano mayor representan realmente el interés de la familia.

Por último, los conflictos, y no necesariamente las maneras de solucionarlos, reflejan los cambios en la forma de vida. Regresamos aquí al último elemento de las prácticas: los discursos. La necesidad de explicar, justificar y atacar discursivamente las prácticas surge de una duda o conflicto, no de paz y concordia, en las situaciones de crisis. En el texto de Elías encontramos dos ejemplos interesantes de discursos críticos sobre las viejas prácticas. En el primer relato, él presenta la crítica a la cual las mujeres someten las prácticas patriarcales de la herencia: "pero igual cuidamos a nuestro padre y nuestra madre". A partir de este discurso, la práctica de la herencia de la tierra en la familia de Elías ha cambiado, porque las mujeres la heredan actualmente de la misma manera y según las mismas reglas que sus hermanos, cambio que no deriva de actos políticos — como la apelación de la primera alcaldesa de Chenalho', Rosa 
Pérez Pérez, al Tribunal Electoral-, sino más bien de un cambio interno, discreto, basado en algo que los familiares de Elías compartían: el principio de reciprocidad. Daniel y sus hermanos pudieron ver un aspecto nuevo de la misma práctica: la contribución de sus hermanas al cuidado de los padres. En este sentido, el cambio de perspectiva es posible sólo cuando se ha aceptado e internalizado la visión alternativa.

Otro ejemplo lo constituyen las opiniones críticas del mismo Elías sobre las decisiones de sus hermanos basadas en el reconocimiento del valor de la tradición. Su hermana Beatriz fue excluida finalmente de la herencia del solar por sus hermanos encabezados por Daniel, quien argumentó que ella, como mujer, no se podía encargar del solar pues esa herencia, según la tradición, le tocó a Benito. Elías no estuvo conforme con ese veredicto: "Pienso que esto es injusto porque él no cuidaba de mi papá". El argumento de Elías parece divorciarse de las reglas tradicionales de la herencia del solar según las cuales éste queda para el hijo menor. Pero tal conclusión sería incorrecta porque la crítica de Elías constituye no un rechazo de la regla de reciprocidad, sino una modificación de ésta. Las nuevas condiciones de vida cambiaron el escenario. El principio de reciprocidad no sólo define los derechos de Benito, el hijo menor de la familia, sino que también asume que la condición sine qua non para la concesión de este derecho es el cumplimiento de un compromiso importante, el cuidado del padre. Pero precisamente este deber no fue cumplido por Benito porque había emigrado a la ciudad para estudiar y vivía y trabajaba fuera de su paraje, mientras que quien se quedó en su lugar en la casa para cuidar al padre fue su hermana Beatriz de manera que, según Elías, a ella se le debía conceder el derecho a heredar el solar. La crítica de Elías, sin embargo, va más allá de esclarecer y modificar la regla de reciprocidad. La expresión "es injusto" cuestiona también la última regla que denominamos el "metaprincipio pragmático-patriarcal". Mientras que antes lo justo e injusto en relación con los conflictos era definido en última instancia por el hermano mayor, actualmente Elías no ve contradicción alguna en decir "la decisión de mi hermano es injusta”. Las prácticas nuevas necesitan discursos nuevos.
Finalmente, queda por aclarar la última cuestión: el papel de los conflictos. Los descritos por Elías son el reflejo de tres fisuras en la forma de vida dominante. En primer lugar, parte de los integrantes de la familia Pasinsa, incluyendo a Elías y a su hermano menor Benito, viven fuera de la comunidad y participan en otra forma de vida, la mestiza, mientras los demás hermanos permanecen en Las Limas. Esta situación causa conflictos que se nutren de una confusión acerca de la forma de participación de los ausentes en el seguimiento de las reglas de herencia, sobre todo de la regla de reciprocidad (relatos 1 y 7 ). La segunda fisura que se abre en la antigua forma de vida tiene que ver con el fenómeno de la migración, que pone en peligro los principios de reciprocidad, de autonomía familiar y de solidaridad interna del linaje (relato 3). Finalmente, la tercera fisura la constituyen los cambios de la posición de la mujer en la cultura indígena (relatos 1 y 2). A estos conflictos se agregan los otros mencionados, sobre todo los derivados de colindancias entre los hermanos, por reparto de bienes o por estrategias internas no aceptadas, es decir, lo que va en contra de los principios de la herencia (relatos 5, 6 y 7 ).

A primera vista parece que estos conflictos hacen la vida de la familia Pasinsa una misión imposible, pero un análisis más profundo, sin embargo, muestra lo contrario dado que, si bien aumenta la conflictividad a partir de las reglas de herencia, también se fortalecen los mecanismos destinados a la solución de los conflictos. En nuestro caso, se observa un mecanismo sólido que hemos llamado "metaprincipio pragmático-patriarcal". En caso de los conflictos que no alcanzan solución, la familia Pasinsa acude a Daniel, de manera que el conflicto se vuelve así un soporte de la autoridad del padre y de su sustituto, el hermano mayor. Es este último quien organiza las reuniones familiares, sostiene la comunicación interfamiliar, presta el espacio para organizar las pequeñas asambleas, da voz a los contendientes, reflexiona e invita a reflexionar. La autoridad de Daniel es innegable, pero no tiránica. Es él quien representa a la familia, quien dialoga con sus hermanos y hermanas para buscar soluciones entre todos. Puede desarrollar su liderazgo gracias a la 
voluntad colectiva de su familia, la pequeña communitas, de manera que puede seguir fortaleciéndose gracias a su liderazgo, el cual no sería posible si no existiese la necesidad de acudir a él. La conclusión simmeliana es clara: los conflictos derivados de las prácticas de herencia en la familia Pasinsa refuerzan y reproducen la familia y, a la postre, el linaje Pasinsa.

\section{Notas}

${ }^{1}$ Una tarea equivale a tres brazadas de ancho por sesenta de largo.

${ }^{2}$ Sus palabras fueron: "Vo'oxuk vinikoxuke ep ta ataik li yosil li jtotike. Jauk li vu'unkutike tz'ebunkutike, muyuk bu takutik me jutebuk. Pero, ko`ol tajk'eltik jtukulantik li jtotik li jmetike. Ko`ol abunjbatik ta abtel li skoltael li jtotik li jmetike k'alal li jchitale; akbunkutik xa ek li osil liee. Ak'o chak'bejbakutik kotolkutik li jtzebunkutike. Li jximnel Elíase, muku’o no'ox ep lavakbeik yosil. Tajchinkutik ta xchakel li osil li'e".

${ }^{3}$ El 19 de julio de 2015 Rosa Pérez Pérez, del Partido Verde, ganó como la primera mujer presidenta de Chenalho' con 8332 votos frente a 7012 votos de su rival del Partido Revolucionario Institucional (PRI). El 1 de octubre tomó protesta en el cargo. En los siguientes meses, los opositores de la alcaldesa no estuvieron de acuerdo con su forma de gobernar y soliitaron su renuncia. Aunque varios hechos violentos en abril y mayo de 2017 le hicieron renunciar en mayo de 2017, el Tribunal Electoral del Poder Judicial de la Federación le ordenó su reinstalación el 17 de agosto del mismo año. Un grupo de individuos armados, simpatizantes de la alcaldesa, retomó la presidencia el día 2 de febrero de 2018. Mientras Moisés Pérez Arias se desempeñaba como juez en el gobierno de Rosa Pérez, las personas de su paraje natal, Chibtik, estaban en contra de la alcaldesa.

\section{Referencias}

Arias, Jacinto (1975). El mundo numinoso de los mayas. México: Secretaría de Educación Pública.

Arias, Jacinto (1985). San Pedro Chenahló. Algo de su historia, cuentos y costumbres. Tuxtla Gutiérrez, Chiapas:
Gobierno del Estado, Instituto Chiapaneco de Cultura.

Barth, Fredrik (1981). "Anthropological Models and Social Reality". En Process and Form in Social Life. Selected Essays of Fredrik Barth, vol. I. Londres: Routledge, pp. 14-31.

Clooney, Francis, X. (2005). "Practices". En William Schweiker (ed.), The Blackwell Companion to Religious Ethics. Australia: Blackwell Publishing, pp. 78-85

Contreras, Ulises (2001). "El conflicto social como generador de padecimiento: litigios por tierra e ilvajinel (mal arrojado por envidia) en Yolonhuitz, Chiapas". En Alteridades, 11(21): 53-64.

Contreras, Ulises (2003). "Conflicto social y enfermedad en los Altos de Chiapas (estudio de caso)". En Estudios Sociológicos, XXI(2), mayo-agosto: 399-420.

Collier, George A. (1976). Fields of Tzotzil: The Ecological Bases of Tradition In Highland Chiapas. Austin: University of Texas Press.

Garza Caligaris, María Ana (2002). Género, interlegalidad y conflicto en San Pedro Chenalhó. México: UNAM/ UNACH.

Garza Caligaris, María Ana (2007). "Conflicto, etnicidad y género en la política interna de San Pedro Chenalhó, Chiapas". En Sociológica, 22(63): 85-110.

Gramsci, Antonio (2009). Pasado y presente. México: Juan Pablos Editor.

Guiteras Holmes, Calixta (1996). Los peligros del alma. Visión del mundo de un tzotzil. México: Fondo de Cultura Económica.

Ixtacuy López, Octavio, Erin I.J. Estrada Lugo y Manuel Roberto Parra Vázquez (2006). “Organización social en la apropiación del territorio: Santa Marta, Chenalhó, Chiapas". En Relaciones. Estudios de Historia y Sociedad, 106(XXVII)

Jacorzynski, Witold (2008). En la cueva de la locura. La aportación de Ludwig Wittgenstein a la antropología social. México: Casa Chata, Porrúa.

Moyal-Sharrock, Danièle (2004). Understanding Wittgenstein's On Certainty. Londres, Nueva York: Palgrave Macmillan.

Schermer Henry y David Jary (2013). Form and Dialectic in Georg Simmel's Sociology. A New Interpretation, Nueva York: Palgrave Macmillan. 
Simmel, Georg (2009). Sociology. Inquiries into the Construction of Social Forms, vol l. Leiden: Brill.

Winch, Peter (1990). Ciencia social y filosofía. Buenos Aires: Amorrortu.
Wittgenstein, Ludwig (1988). Investigaciones filosóficas. Barcelona: Crítica.

Wittgenstein, Ludwig (1997). Sobre la certeza. Barcelona: Gedisa. 